\title{
Einige Bemerkungen über die Wirkung fortgesetzter Bogenlampenlichtbäder auf die Haut (und ihre therapeutische Verwendung).
}

\author{
Von \\ K. A. Heiberg.
}

(Aus Finsens medicinske Lysinstitut, Kopenhagen.)

Die Fragen, die sich an die Lichtveränderungen der Haut bei langdauernder Behandlung mit Kohlenbogenlichtbädern knüpfen, sind sehr in den Vordergrund getreten, da die in den letzten Jahren bei der Tuberkulosebehandlung mit universellem Bogenlampenlicht erzielten Erfolge seine ausgedehnte Anwendung mit herbeigeführt haben'1).

In folgendem wollen wir uns mit der Wirkung der Strahlen auf die Oberhaut beschäftigen, zu deren Studium wir eine Anzahl von Untersuchungen angestellt haben, die 25 Excisionen umfassen; die Excisionen wurden $5 \mathrm{~cm}$ medial vom Trochanter major vorgenommen, an einer infolge der Schrägwirkung übrigens nicht sonderlich stark belichteten Stelle.

Die Basalzellenschicht ist jene Schicht, in der das Wachstum und die Neubildung des Epithels stattfindet. Normalerweise geschieht die Kernteilung nur hier, und von hier aus entwickeln sich die Epithelzellen, um später ihre verschiedenen Veränderungen bis zum Stratum corneum zu durchlaufen. Die Basalzellenschicht ist allein imstande, sich fortzupflanzen, und sie allein enthält bei Weißen die feineren oder gröberen Pigmentkörner. Findet man auch in den höheren Schichten Pigment, so spricht man bei der weißen Rasse von Überpigmentierung.

Daß das gewöhnliche in den basalen Epithelzellschichten vorhandene Pigment sich in den höheren Epithelschichten nicht hält, kann, nach Unnas Untersuchungen mit der Rongalitweißmethode, durch die Verteilung des Sauerstoffs in der Haut erklärt werden. Die Menge Sauerstoff, die für das Vorhandensein des Pigments in Epithel notwendig ist, ist nur in der Basalschicht (Keimschicht) nachzuweisen, und an diese Oxydationsstätte mit ihrem Uberschuß an. Sauerstoff ist das Pigment in überwiegendem Maße gebunden. Wenn, wie bei der weißen Rasse, nur eine geringe Pigmentmenge gebildet wird, so werden „die

1) Vgl. Reyn und Ernst, Die Resultate der Behandlung mit künstlichen chemischen Lichtbädern bei Lupus vulgaris und chirurgischer Tuberkulose. Strahlentherapie 10. 1919. - Strandberg, Die Anwendung des universellen Lichtbades bei rbinolaryngologischer Tuberkulose. Strahlentherapie 10. 1919. 
K. A. Heiberg: Bemerk. über d. Wirkung fortges. Bogenlampenlichtbäder usw. 307

älteren Zellen im Stratum spinocellulare und die Zellen der Hornschicht, reduzierende Kraft genug haben, um es zu vernichten: an diesen reduzierenden Stellen kann es nur dann erhalten bleiben, wenn, wie bei den Negern, ein ganz besonders großer Pigmentreichtum gegeben ist.

Unter der Einwirkung der Kohlenbogenlichtbäder tritt nun eine Vermehrung des Pigmentes in der Basalzellenschicht ein, und zwar hat sich gezeigt, daß es während der Lichtbehandlung selbst überall im Protoplasma der Zellen verteilt ist, wohingegen es einige Zeit danach sich nur in der oft beschriebenen Anordnung am distalen Kernpol findet (in. 2 Fällen nachgewiesen). Der größere Pigmentreichtum der Basalzellen während der Kohlenbogenlichtbehandlung fübrt natürlich auch einen gewissen Pigmentgehalt der höheren Zellschichten des Stratum Malpighi herbei, doch ist diese Wirkung keine andauernde, wie überhaupt die Pigmentierung bald nach Aussetzen der Behandlung schwindet.

Die durch die Lichtbehandlung hervorgerufene vermehrte Pigmentbildung stellt also für die Dauer derselben eine Schutzmaßnahme dar, und ist in dieser Hinsicht leistungsfähiger als die natürliche Hyperpigmentierung. Denn $W$ ith $^{\mathbf{1}}$ ) konnte zeigen, daß die sehr kräftige Pigmentierung bei einem Neger geringere Widerstandsfähigkeit gegen das Licht zeigte als die minder kräftige, wie sie durch Lichtbäder erworben wird. Er wies aber gleichzeitig auch darauf hin, daß die Rolle des Pigments als Schutz nicht zu überschätzen sei und daß es vor allem nicht den einzigen Schutz darstellt, denn bei Vitiligo z. B. müssen augenscheinlich andere Schutzmittel gegen die langdauernde Behandlung mit Bogenlichtbädern in Tätigkeit treten.

Auch wir haben neben der Pigmentierung noch andere Faktoren gefunden, die neben dem Pigment das ihrige dazu beitragen, die lichtbehandelte Haut undurchsichtiger zu machen. Denn die chronische Lichtwirkung auf die Oberhaut durch universelle Bogenlampenbäder ist durch Betrachtung der Pigmentbildung nicht erschöpft, wie im allgemeinen angenommen wird, sondern es ist noch auf ein Moment, das gleichfalls in der Basalzellschicht seinen Ausgangspunkt hat, hinzuweisen, und das ist das ständig vor sich gehende stärkere Zellenwachstum, das sich schon klinisch durch die stetig fortgesetzte, stärker als normale Abschlieferung und Erneuerung der Hornschicht zu erkennen gibt.

Bei diesem fortgesetzten Wachstumsprozesse steht man sicher auch einem Moment gegenüber, dessen Bedeutung als Lichtreaktion in Betracht gezogen werden muß. Busck und Hasselbalch haben auf den Einfluß hingewiesen, den die Dicke der Epidermis auf die Licht-

1) Vgl. Carl With; Studien über Lichtwirkung bei Vitiligo. Hospitalstidende 1920 . 
308 K. A. Heiberg: Bemerk. iber d. Wirkung fortges. Bogenlampenlichtbäder usw.

absorption hat. Der größere Zellreichtum (dichter gestellte Kerne!) dürfte aber in Betracht kommen nebea einer geringeren Vermehrung in der Höhe, die allerdings in diesem Zusammenhang auch nicht ausgeschlossen werden kann.

Mikroskopisch sieht man nämlich nach fortgesetzten Bogenlichtbädern, wenn auch nur in geringerem Grad, eine Wiederholung des Bildes, das die Oberhaut in der ersten Zeit nach der Belichtung bietet. Von den akuten, entzündungsartigen, sofortigen Veränderungen soll ja hier nicht weiter die Rede sein, man sieht aber auch hier, daß die Dicke der Zellschichten vermehrt ist — was allerdings bei einem so variablen Organ, wie es die Epidermis darstellt, schwierig festzustellen ist - vor allem aber sind in den dicht gestellten Zellschichten, besonders nach unten hin, die Zellen zahlreicher als sonst und stehen dichter nebeneinander.

Die Hornschicht ist verbreitert und die Keratohyalinschicht (Stratum granulosum) körniger und auch mehr ohne Unterbrechung fortlaufend, wie sich dies bekanntlich auch normalerweise an Stellen, wo die Erneuerung der Haut eine lebhafte ist, findet.

Wenn auch in einigen Fällen, wie bei der Pigmentierung durch mechanische Einwirkung, ein Zusammenhang zwischen Wachstum und Pigmentierung vorhanden sein kann, so sind diese Vorgänge als gleichgeordnete, auf jeden Fall voneinander durchaus nicht abhängige Phänomene aufzufassen.

Die stärkste Reaktion findet șich natürlich zu Anfang der Behandlang, und klingt bei fortgesétzter Behandlung in dem Maße ab, als die eingetretene Pigmentierung und das stärkere Zellwachstum die Haut dauernd erneuert und dadurch ihre Schichten widerstandsfähiger und undurchsichtiger macht.

Berücksichtigt man dies Verhalten bezüglich des Wachstums, so erhält man einesteils Finblick in den ganzen umfassenden Prozeß, der unter der chronischen Lichtwirkung vor sich geht — handelt es sich doch um eine sich immer wiederholende Erneuerung der Epidermis, deren Gewicht ungefähr $1 / 2 \mathrm{~kg}$ beträgt, andernseits versteht man auch, weshalb die universellen Bogenlichtbäder als Hilfsmittel bei verschiedenen Hautkrankheiten angewendet werden können, bei denen die Erneuerung der Epidermis eine Rolle spielt.

\section{Zusammenfassung.}

Bei der Behandlung mit Kohlenbogenlicht kommt es zu einer Hauterneuerung, und zwar nicht allein zu Beginn der Lichtbäder - wenn auch da natürlich am kräftigsten - sondern auch späterhin. 